\title{
A DIAMETER PINCHING SPHERE THEOREM FOR POSITIVE RICCI CURVATURE
}

\author{
JYH-YANG WU
}

(Communicated by Jonathan Rosenberg)

\begin{abstract}
In this note we generalize Shiohama's volume pinching sphere theorem to a diameter pinching sphere theorem for positive Ricci curvature.
\end{abstract}

\section{INTRODUCTION}

In this paper a manifold $M$ always means a complete connected Riemannian manifold of dimension $n$ and $v(M)$ will denote the volume of $M, d(M)$ the diameter of $M, K_{M}$ the sectional curvature of $M$ and $\mathrm{Ric}_{M}$ the Ricci curvature of $M$.

The sphere theorem due to Klingenberg [K] says that if $M$ is a complete, simply connected $n$-dimensional manifold with $1 / 4<K_{m} \leq 1$, then $M$ is homeomorphic to the $n$-sphere $S^{n}$. In 1977, Grove and Shiohama [GS] proved the generalized sphere theorem which states that a complete $n$-manifold $M$ with $K_{M} \geq 1$ and $d_{M}>\pi / 2$ is homeomorphic to $S^{n}$.

An elegant theorem due to Myers [M] states that if the Ricci curvature of a complete $n$-manifold $M$ satisfies that $\mathrm{Ric}_{M} \geq n-1$, then $d(M) \leq \pi$ and hence $M$ is compact and its fundamental group $\pi_{1}(M)$ is finite.

In [C], S. Y. Cheng proved the Maximal Diameter Sphere Theorem which states that if $\mathrm{Ric}_{M} \geq n-1$ and $d_{M}=\pi$, then $M$ is isometric to the standard sphere $S^{n}$. Naturally one will ask if there is a $d_{n}<\pi$ which depends only on $n$ such that if $\operatorname{Ric}_{M} \geq n-1$ and $d(M)>d_{n}, M$ is homeomorphic to $S^{n}$. Since we can find metrics on $M=S^{j} \times S^{j}$ so that $\mathrm{Ric}_{M}=2 j-1$ and the diameter approaches $\pi$ as $j$ goes to $\infty$, for the Ricci curvature case, the dependence on $n$ at least seems inevitable.

This problem is still open. However with some more restrictions on $M$, Shiohama $[\mathrm{S}]$ showed the following volume pinching sphere theorem:

Received by the editors December 18, 1988.

1980 Mathematics Subject Classification (1985 Revision). Primary 53C20.

Key words and phrases. Ricci curvature, geodesic ball, contratibility radius. 
Theorem. Let $n$ be a positive integer and let $\kappa>0$ be a constant. Then there exists an $\varepsilon(n, \kappa)>0$ such that if $M$ is an $n$-dimensional complete manifold with $\mathrm{Ric}_{M} \geq n-1, K_{M} \geq-\kappa^{2}$ and $v(M) \geq \alpha(n, \pi-\varepsilon(n, \kappa))$, then $M$ is homeomorphic to $S^{n}$, where $\alpha(n, r)$ is the volume of the r-ball on $S^{n}(1)$.

In this note we generalize this result to the following diameter pinching sphere theorem:

Main Theorem. Let $n$ be a positive integer and let $\kappa>0, r \in(\pi / 2, \pi)$. Then there is $\delta$ depending only on $n, \kappa$ and $r$ such that if $M$ is an $n$-dimensional complete manifold with $\mathrm{Ric}_{M} \geq n-1, K_{M} \geq-\kappa^{2}, v(M) \geq \alpha(n, r)$ and $d(M)>\pi-\delta, M$ is homeomorphic to $S^{n}$.

The author does not know if the assumption for the sectional curvature and the volume is essentially needed. Under this assumption the radius of contractible metric balls can be bounded away from 0 . In our proof we show that if the diameter of $M$ is close to $\pi$, the contractibility radius of two particular points which realize the diameter of $M$ will be also close to $\pi$. Then we will be able to cover $M$ with two contractible metric balls and appeal to the generalized Schoenflies theorem to complete the proof, where the Generalized Schoenflies Theorem [B] states that if $M$ is covered by two open disks, then $M$ is homeomorphic to $S^{n}$.

\section{ESTIMATE OF CONTRACTIBILITY RADIUS}

This section is essentially based on the paper [S] of Shiohama. Let $M$ be an $n$-dimensional manifold. For a fixed point $x \in M$ consider the distance function $d_{x}: M \rightarrow R, d_{x}(y)=d(x, y)$. A point $y \in M$ is called a critical point of $d_{x}$ if for any nonzero tangent vector $u \in T M_{y}$, there is a minimizing geodesic from $y$ to $x$ whose tangent vector at $y$ makes an angle with $u$ not greater than $\pi / 2$. Hence a critical point of $y$ of $d_{x}$ belongs to the cut locus $C_{x}$ of $x$.

The contractible radius $c(x)$ at $x \in M$ is defined as

$$
c(x)=\operatorname{supp}\left\{r: \bar{B}_{r}(x) \text { is contractible to } x\right\} .
$$

The following two lemmas can be found in [S]:

Lemma 2.1. For any $x \in M$, if $\bar{B}_{r}(x)$ contains no critical point of $d_{x}$ except at the origin $x$ of the ball, then $\bar{B}_{r}(x)$ is contractible to $x$. In other words, $c(x)$ is not less than the positive minimum critical value $:=c_{1}(x)$ of $d_{x}$.

Lemma 2.2. Let $\varepsilon$ be in $(0, \pi)$. Assume that $\operatorname{Ric}_{M} \geq n-1$ and $v(M) \geq$ $\alpha(n, \pi-\varepsilon)$. For every point $x \in M$ and a number $\theta \in(0, \pi)$ and for every unit tangent vector $u \in S M_{x}$ let $\Gamma(u, \theta)=\left\{w \in T M_{x}: \varangle(u, w)<\theta\right\}$. Then there exists a positive smooth function $r \rightarrow \theta(r, n, \varepsilon), 0<r<\pi-\varepsilon$ such that if every $w \in \Gamma(u, \theta) \cap C_{x}$ has norm $\|w\| \leq r$, then $\theta \leq \theta(r, n, \varepsilon) . \theta(r, n, \varepsilon)$ is 
obtained as the solution of

$$
c_{n-2} \int_{0}^{\theta(r, n, \varepsilon)} \sin ^{n-2} t d t \int_{r}^{\pi} \sin ^{n-1} t d t=\alpha(n, \varepsilon),
$$

where $c_{m}$ is the volume of $S^{m}(1)$.

Remark. For $\delta \in(\varepsilon, \pi / 2), \theta(\pi-2 \delta, n, \varepsilon)<\pi / 2$.

We are now in a position to estimate $c_{1}(x)$.

Theorem 2.3. Let $n$ be a positive integer and let $\kappa \geq 0$ and $\varepsilon \in(0, \pi / 2)$ be given. Then there exists for a fixed number $\delta \in(\varepsilon, \pi / 2)$ a constant $c_{\delta}(n, \kappa, \varepsilon)>$ 0 such that if $M$ is a complete $n$-dimensional manifold with

$$
\operatorname{Ric}_{M} \geq n-1, \quad K_{M} \geq-\kappa^{2}, \quad v(M) \geq \alpha(n, \pi-\varepsilon),
$$

Then $c_{1}(x) \geq c_{\delta}(n, \kappa, \varepsilon)$ for every point $x \in M$. The constant is given by

$$
c_{\delta}(n, \kappa, \varepsilon)=\min \left\{\pi-2 \delta, \kappa^{-1} \tanh ^{-1}[\tanh (\pi-2 \delta) \kappa \cos \theta(\pi-2 \delta, n, \varepsilon)]\right\} .
$$

Proof. Let $r_{1}=\pi-2 \delta$ and let $x \in M$ be a fixed point and $y$ a critical point of $d_{x}$ with the positive minimum critical value $r_{0}=c_{1}(x)$. Let $u \in S M_{x}$ be the unit vector tangent to a minimizing geodesic $\gamma_{u}:\left[0, r_{0}\right] \rightarrow M$ with $\gamma_{u}(0)=x$, $\gamma_{u}\left(r_{0}\right)=y$.

By the above lemma and the continuity of the map $w \in S M_{x} \rightarrow$ the distance from $x$ to the cut point of $x$ along the geodesic $t \rightarrow \exp _{x} t w$, there is a $w \in S M_{x}$ with the properties $\varangle(u, w) \leq \theta\left(r_{1}, n, \varepsilon\right)$ and $\gamma_{w}$ has the cut point to $x$ along it at $\gamma_{w}\left(t_{1}\right)$ with $t_{1} \geq r_{1}$. If $r_{0} \geq r_{1}$, then we are done. Hence we can assume that $r_{0}<r_{1}$. The Toponogov Comparison Theorem implies that if $\alpha=\varangle(u, w)$ and if $r_{2}=d(y, z)$ where $z=\gamma_{w}\left(t_{1}\right)$, then

$$
\cosh r_{2} \kappa \leq \cosh t_{1} \kappa \cosh r_{0} \kappa-\sinh t_{1} \kappa \sinh r_{0} \kappa \cos \alpha .
$$

Since $y$ is a critical point of $d_{x}$, there is, for a minimizing geodesic from $y$ to $z$, a minimizing geodesic from $y$ to $x$ (possibly different from $\gamma_{u}$ ) whose angle at $y$ is not greater than $\pi / 2$. Thus again by the Toponogov Comparison Theorem, one has

$$
\cosh t_{1} \kappa \leq \cosh r_{0} \kappa \cosh r_{2} \kappa .
$$

Eliminate $r_{2}$ from the above inequalities to obtain

$$
\cosh t_{1} \kappa \tanh r_{0} \kappa \geq \cos \alpha .
$$

Insert $\alpha \leq \theta\left(r_{1}, n, \varepsilon\right)<\pi / 2$ and $t_{1} \geq r_{1}=\pi-2 \delta$ to complete the proof.

Remark. This theorem is basically due to Shiohama. In [S], he proved this for $\varepsilon \in(0, \pi / 3)$. 


\section{The PROOF OF MAIN TheOREM}

Before we start to prove the main theorem let's recall the Bishop-Gromov Volume Comparison Theorem [G].

Let $M$ be a complete manifold of dimension $n$ with $\operatorname{Ric}_{M} \geq-(n-1) \kappa^{2}$, where $\kappa$ is a real or a pure imaginary number. Let $M\left(-\kappa^{2}\right)$ be the complete simply connected $n$-dimensional space form of constant sectional curvature $-\kappa^{2}$. For a point $x \in M$ and for an $r>0$ let $B_{r}(x)$ be the metric $r$-ball centered at $x$. A metric $r$-ball in $M\left(-\kappa^{2}\right)$ is denoted by $\widetilde{B}_{r}$. With these notations the Bishop-Gromov Volume Comparison Theorem is stated as

Lemma 3.1. For any fixed $x \in M$ and $0 \leq r \leq R$,

$$
\frac{v\left(B_{r}(x)\right)}{v\left(B_{R}(x)\right)} \geq \frac{v\left(\widetilde{B}_{r}\right)}{v\left(\widetilde{B}_{R}\right)} .
$$

Let $\left\{M_{k}\right\}$ be a sequence of complete manifolds with $\mathrm{Ric}_{M_{k}} \geq n-1, K_{M_{k}} \geq$ $-\kappa^{2}$ and $v\left(M_{k}\right) \geq \alpha(n, r)$ where $\kappa, r$ are as in the Main Theorem and assume that $d_{k}=d\left(M_{k}\right) \rightarrow \pi$ as $k \rightarrow \infty$.

Let $p_{k}$ and $q_{k}$ be in $M_{k}$ with $d\left(p_{k}, q_{k}\right)=d_{k}$. Now we are going to investigate the contractibility radius of $p_{k}$ and $q_{k}$. Choose $y_{k} \in M_{k}$ to be a critical point of $d_{p_{k}}$ with the positive minimum critical value $r_{k}=c_{1}\left(p_{k}\right)$ and let $t_{k}=d\left(y_{k}, q_{k}\right)$. By Theorem 2.3, $r_{k} \geq r_{0}$ for some positive number $r_{0}$. Without loss of generality, we can assume that $\lim r_{k}=\alpha \geq r_{0}$ and $\lim t_{k}=\beta$. Since $r_{k}+t_{k} \geq d_{k}$, $\alpha+\beta \geq \pi$.

Claim 1. $\beta=\pi-\alpha$.

Proof. Supposing this is not true, one can find a positive number $\varepsilon<$ $1 / 4 \min \left\{r_{0}, \alpha+\beta-\pi\right\}$ and $N_{0}$ such that if $k \geq N_{0}$, then $t_{k} \geq \pi-\alpha+3 \varepsilon$ and $\alpha-\varepsilon<r_{k}<\alpha+\varepsilon$. Hence $t_{k}>d_{k}-r_{k}+2 \varepsilon$. Thus the balls $B_{p_{k}}\left(r_{k}-\varepsilon\right)$, $B_{q_{k}}\left(d_{k}-r_{k}+\varepsilon\right)$ and $B_{y_{k}}(\varepsilon)$ are pairwise disjoint in $M_{k}$. This gives

$$
v\left(M_{k}\right) \geq v\left(B_{p_{k}}\left(r_{k}-\varepsilon\right)\right)+v\left(B_{q_{k}}\left(d_{k}-r_{k}+\varepsilon\right)\right)+v\left(B_{y_{k}}(\varepsilon)\right) .
$$

Dividing by $v\left(M_{k}\right)$ on both sides and using the Bishop-Gromov Volume Comparison Theorem, one has

$$
1 \geq\left[\alpha\left(n, r_{k}-\varepsilon\right)+\alpha\left(n, d_{k}-r_{k}+\varepsilon\right)+\alpha(n, \varepsilon)\right] / \alpha(n, \pi) .
$$

Now letting $k \rightarrow \infty$,

$$
1 \geq 1+\alpha(n, \varepsilon) / \alpha(n, \pi) .
$$

This is impossible, hence $\beta=\pi-\alpha$.

Claim 2. $\alpha=\pi$.

Proof. Since $y_{k}$ is a critical point of $d_{p_{k}}$ there exists, for a minimizing geodesic from $y_{k}$ to $q_{k}$ a minimizing geodesic from $y_{k}$ to $p_{k}$ whose angle at $y_{k}$ is not 
greater than $\pi / 2$. Thus the Toponogov Comparison Theorem applies for this triangle to give

$$
\cosh d_{k} \kappa \leq \cosh r_{k} \kappa \cosh t_{k} \kappa
$$

Letting $k \rightarrow \infty$ and using Claim 1 ,

$$
\cosh \pi \kappa \leq \cosh \alpha \kappa \cosh (\pi-\alpha) \kappa .
$$

This gives that $\alpha=\pi$.

The Proof of Main Theorem. Suppose that Main Theorem is false. Then there exists a sequence of manifolds $M_{k}$ which are not homeomorphic to $S^{n}$ such that $\operatorname{Ric}_{M_{k}} \geq n-1, K_{M_{k}} \geq-\kappa^{2}, v\left(M_{k}\right) \geq \alpha(n, r)$ and $d_{k}=d\left(M_{k}\right) \rightarrow \pi$. Let $p_{k}$ and $q_{k}$ be in $M_{k}$ with $d\left(p_{k}, q_{k}\right)=d_{k}$. By the above argument and Lemma 2.1 , the contractibility radii $c\left(p_{k}\right)$ and $c\left(q_{k}\right)$ are greater than $2 \pi / 3$ for large $k$.

The minimal radius $R_{k}$ of closed balls around $p_{k}$ and $q_{k}$ by which $M_{k}$ is covered satisfies $d_{k} / 2 \leq R_{k} \leq d_{k}$ and $R_{k}=\max \left\{d\left(p_{k}, x\right): x \in M_{k}\right.$, $\left.d\left(p_{k}, x\right)=d\left(x, q_{k}\right)\right\}$. If $x_{k} \in M_{k}$ is a point with $d\left(p_{k}, x_{k}\right)=d\left(x_{k}, q_{k}\right)=R_{k}$, then

$$
v\left(M_{k}\right) \geq v\left(B_{p_{k}}\left(d_{k} / 2\right)\right)+v\left(B_{q_{k}}\left(d_{k} / 2\right)\right)+v\left(B_{x_{k}}\left(R_{k}-d_{k} / 2\right)\right) .
$$

Dividing by $v\left(M_{k}\right)$ and again using the Bishop-Gromov Volume Comparison Theorem ,

$$
1 \geq\left[2 \alpha\left(n, d_{k} / 2\right)+\alpha\left(n, R_{k}-d_{k} / 2\right)\right] / \alpha(n, \pi) .
$$

Since $d_{k} \rightarrow \pi$, we conclude that $R_{k} \rightarrow \pi / 2$. Hence for large $k, R_{k}<2 \pi / 3$. Therefore for large $k, M_{k}$ can be covered by two contractible metric balls $B_{p_{k}}(2 \pi / 3)$ and $B_{q_{k}}(2 \pi / 3)$. The Generalized Schoenflies Theorem implies that $M_{k}$ is homeomorphic to $S^{n}$. This desired contradiction completes the proof.

\section{ACKNOWLEDGMENT}

I would like to thank K. Shiohama whose work is used extensively throughout this paper.

\section{REFERENCES}

[B] M. Brown A proof of the generalized Schoeflies theorem, Bull. Amer. Math. Soc. 66 (1960), 74-76.

[BC] R. Bishop and R. L. Crittendon, Geometry of manifolds, Academic Press, New York, 1974.

[C] S. Y. Cheng, Eigenvalue comparison theorem and its geometric applications, Math. Zeit. 143 (1975), 289-297.

[CE] J. Cheeger and D. Ebin, Comparison theorems in Riemannian geometry, North-Holland, Amsterdam and New York, 1975.

[G] M. Gromov, Curvature, diameter and Betti numbers, Comment. Math. Helv. 56 (1981), 179-195.

[GS] K. Grove and K. Shiohama, A generalized sphere theorem, Ann. of Math. (2) 106 (1977), 201-211. 
[I] Y. Itokawa, The topology of certain Riemannian manifolds with positive Ricci curvature, J. Differential Geom. 18 (1983), 151-156.

[K.] W. Klingenberg, Über Riemannsche Mannigfaltigkeiten mit positiver Krümmung, Comment. Math. Helv. 35 (1961), 47-54.

[M] S. B. Myers, Riemannian manifolds with positive mean curvature, Duke Math. J. 8 (1941), 401-404.

[S] K. Shiohama, A sphere theorem for manifolds of positive Ricci curvature, Trans. Amer. Math. Soc. 275 91983), 811-819.

Department of Mathematics, University of Chicago, 5734 South University Avenue UNIVERSITY, ChICAGO, ILLINOIS 60637 